\title{
Existence of Kähler-Einstein metrics and multiplier ideal sheaves on del Pezzo surfaces
}

\author{
Gordon Heier
}

Received: 31 March 2008 / Accepted: 30 December 2008 / Published online: 19 February 2009

(C) The Author(s) 2009. This article is published with open access at Springerlink.com

\begin{abstract}
We apply Nadel's method of multiplier ideal sheaves to show that every complex del Pezzo surface of degree at most six whose automorphism group acts without fixed points has a Kähler-Einstein metric. In particular, all del Pezzo surfaces of degree 4, 5, or 6 and certain special del Pezzo surfaces of lower degree are shown to have a Kähler-Einstein metric. These existence statements are not new, but the proofs given in the present paper are less involved than earlier ones by Siu, Tian and Tian-Yau.
\end{abstract}

Mathematics Subject Classification (2000) $\quad 14 J 26 \cdot 14 \mathrm{~J} 45 \cdot 32 \mathrm{Q} 20$

\section{Introduction}

Let $X$ be a compact complex manifold. It is said to be a Kähler-Einstein manifold if there exists a Kähler form $\omega=\frac{i}{2} \sum_{i, j} g_{i} \bar{j} d z_{i} \wedge d \bar{z}_{j}$ such that $\omega$ and its Ricci curvature form are proportional, i.e.,

$$
\operatorname{Ricci}(\omega)=-i \partial \bar{\partial} \log \operatorname{det}\left(g_{i} \bar{j}\right)=\lambda \omega
$$

for some $\lambda \in \mathbb{R}$. The hermitian metric $\sum_{i, j} g_{i j} d z_{i} \otimes d \bar{z}_{j}$ corresponding to such an $\omega$ is called a Kähler-Einstein metric. Since the anticanonical class $c_{1}\left(-K_{X}\right)$ (which is also referred to as the first Chern class $\left.c_{1}(X)\right)$ is known to always contain the form $\frac{1}{2 \pi} \operatorname{Ricci}(\omega)$ by a result of Chern, Kähler-Einstein metrics can only exist on manifolds with definite or vanishing anticanonical class.

In his ground-breaking work [35,36], Yau proved Calabi's conjecture, which says that on a compact Kähler manifold, for any given Kähler class, every form representing $2 \pi c_{1}(X)$ can be obtained as the Ricci curvature form of a unique Kähler form in the given Kähler

G. Heier $(\square)$

Department of Mathematics, University of California, Riverside,

900 University Avenue, Riverside, CA 92521, USA

e-mail: heier@math.ucr.edu 
class. As a corollary, on a Kähler manifold with vanishing first Chern class, to every Kähler class there corresponds a unique Ricci flat Kähler-Einstein metric.

On compact complex manifolds with negative anticanonical line bundle, the existence of Kähler-Einstein metrics has been established independently by Aubin [1,2] and Yau (op. cit.). In the case of positive anticanonical line bundle, a Kähler-Einstein metric may or may not exist. Different obstructions have been formulated by Matsushima [22], Futaki [11] and others (see [4] for a nice survey).

In the mid 1980s, Siu [28], Tian [29] and Tian and Yau [33] provided sufficient conditions for the existence of Kähler-Einstein metrics in the case of positive anticanonical bundles, settling certain cases (see below). However, these results were not strong enough to completely clarify the question of existence of Kähler-Einstein metrics on del Pezzo surfaces. Recall that del Pezzo surfaces are two-dimensional compact complex manifolds with positive anticanonical line bundle. The classification of del Pezzo surfaces tells us that they are isomorphic either to $\mathbb{P}^{1} \times \mathbb{P}^{1}, \mathbb{P}^{2}$, or $\mathbb{P}^{2}$ blown up in $r \in\{1, \ldots, 8\}$ general points (see Sect. 2 ).

For $\mathbb{P}^{1} \times \mathbb{P}^{1}$ and $\mathbb{P}^{2}$, the existence of a Kähler-Einstein metric follows directly from the fact that the Fubini-Study metric on $\mathbb{P}^{1}$ and $\mathbb{P}^{2}$ is Kähler-Einstein. For $\mathbb{P}^{2}$ blown up at one or two points, one can show that the so-called Futaki-invariant does not vanish and therefore obstructs the existence of a Kähler-Einstein metric in these cases (see [32, Examples 3.10, 3.11] for a nice discussion). Alternatively, and more in the spirit of this paper, it suffices to observe that the automorphism groups are non-reductive Lie groups, which precludes the existence of Kähler-Einstein metrics according to Matsushima. For the unique del Pezzo surface coming from the blow up of three points, the existence of a Kähler-Einstein metric was shown by [28] and, independently, by the combined efforts of [29,33]. For the unique del Pezzo surface coming from the blow up of four points, the existence of a Kähler-Einstein metric was shown by $[29,33]$. For the general case of del Pezzo surfaces coming from the blow up of $r \in\{5, \ldots, 8\}$ points, Tian's paper [30] finally gave a proof of the fact that they do carry Kähler-Einstein metrics. However, the proof certainly is not short in length.

Remark 1.1 In the paper [33], the authors apparently overlooked the fact that every del Pezzo surface coming from the blow up of five points in $\mathbb{P}^{2}$ can be obtained as the intersection of quadrics in $\mathbb{P}^{4}$ of the form

$$
\sum_{i=0}^{4} X_{i}^{2}=\sum_{i=0}^{4} a_{i} X_{i}^{2}=0,
$$

where $a_{i} \neq a_{j}$ for $i \neq j$. This fact follows, for example, from [13, p. 551] together with the Normal Form Lemma for Simple Pencils of Quadrics [15, Lemma 22.42]. Therefore, the arguments in [33, pp. 188-189] actually settle the case $r=5$ completely, and the belowmentioned dichotomy is already present in the work of Tian and Yau.

A different approach to the problem of existence of Kähler-Einstein metrics was taken by Nadel in his seminal paper [25]. In that paper, Nadel introduces the notion of a multiplier ideal subsheaf of the sheaf of holomorphic functions, partly motivated by the successful use of a similar concept in Kohn's paper [19] on boundary regularity for the complex Neumann problem on weakly pseudoconvex domains of finite type. Nadel proves that the non-existence of certain multiplier ideal sheaves is a sufficient condition for the existence of Kähler-Einstein metrics on a given Fano manifold.

The purpose of this paper is to answer the following open question: To what extent can the technique of multiplier ideal sheaves be used to prove the existence of Kähler-Einstein metrics on del Pezzo surfaces? It turns out that there is not a uniform answer to this question 
due to a dichotomy between the case of at most five points blown up and the case of at least six points blown up. The precise main theorem we prove is the following.

Main Theorem 1.2 Let $X$ be a del Pezzo surface obtained by blowing up $\mathbb{P}^{2}$ in 3, 4, or 5 points. Then the statement in the conclusion of Theorem 3.3 ("for all compact $G \subseteq \operatorname{Aut}(X)$ ), the line bundle $-K_{X}$ possesses...”) is false. In particular, there exists a Kähler-Einstein metric on $X$.

Naturally, the reader may wonder what happens for six or more points blown up. Due to the nature of our proof based on automorphism groups, we find it impossible to prove the analogous statement for 6,7 , or 8 points using our method. However, it turns out that there is a previous result of Nadel that addresses precisely the case of 6, 7, or 8 points blown up:

Theorem 1.3 [26] Let $X$ be a del Pezzo surface obtained by blowing up $\mathbb{P}^{2}$ in 6, 7, or 8 points. Let the automorphism group of $X$ act without fixed points on $X$. Then $X$ carries a Kähler-Einstein metric.

Note that Nadel's result is complementary to ours in the sense that it requires six or more points in an essential way to work. For the sake of completeness, we briefly reproduce Nadel's proof of Theorem 1.3 in Sect. 7, filling in some minor missing details in the proof of Proposition 7.3. The question regarding the applicability of Theorem 1.3 (e.g., to the cubic Fermat hypersurface in $\mathbb{P}^{3}$ ) is discussed at the end of Sect. 7.

Finally, we would like to point out that the proofs of Theorems 1.2 and 1.3 actually yield a stronger result. It follows straight from the definitions that, for a compact subgroup $G \subseteq \operatorname{Aut}(X)$, the fact that Tian's invariant $\alpha_{G}(X)$ (see [29]) is less than 1 implies the existence of a certain non-trivial $G$-invariant multiplier ideal sheaf. However, it is precisely the existence of such a multiplier ideal sheaf that is being ruled out in the proofs below. Therefore, it is immediate that we actually prove the following Theorem, which implies the existence of Kähler-Einstein metrics by the methods of [29].

Theorem 1.4 Let $X$ be a del Pezzo surface as described in the assumptions of either Theorem 1.2 or 1.3 . In the case of three points blown up, let $G=\left(\mathbb{Z}_{2} \times \mathbb{Z}_{2}\right) \rtimes\left(S_{3} \times \mathbb{Z}_{2}\right) \subset \operatorname{Aut}(X)$; in the remaining cases, let $G=\operatorname{Aut}(X)$. Then $\alpha_{G}(X) \geq 1$.

To the author's knowledge, this result has been unknown at least in the case of 4 points. It has been known for some time now (see [6]) that the $\alpha$-invariant coincides with the $\log$ canonical threshold, so in fact this is a new algebraic geometric statement.

\section{Classification and basic properties of del Pezzo surfaces}

Definition 2.1 A del Pezzo surface is a two-dimensional compact complex manifold $X$ whose anticanonical line bundle $-K_{X}$ is ample. We call the self-intersection number $\left(-K_{X}\right)^{2}=K_{X}^{2}$ the degree of $X$. We will denote the degree also by $d_{X}$.

We now gather some important facts about del Pezzo surfaces, resulting in the standard classification (see [5, 14,23]).

Facts 2.2 For every del Pezzo surface $X$, the Picard group Pic $X$ satisfies

$$
\text { rank Pic } X+d_{X}=10 \text {. }
$$

In particular, $d_{X} \leq 9$. 
If $d_{X}=9$, then $X$ is isomorphic to $\mathbb{P}^{2}$.

If $d_{X}=8$, then $X$ is isomorphic either to $\mathbb{P}^{1} \times \mathbb{P}^{1}$ or to $\tilde{\mathbb{P}}^{2}$, i.e., $\mathbb{P}^{2}$ blown up at one point.

If $7 \geq d_{X} \geq 1$, then $X$ is isomorphic to $\mathbb{P}^{2}$ blown up at $r=9-d_{X}$ points which have the following properties:

(i) no three points lie on a line,

(ii) no six points lie on a conic,

(iii) no seven points lie on a cubic such that the eighth is a double point of the cubic.

Any set of $r=9-d_{X}$ points satisfying the above three properties will be said to be in general position, and, conversely, the result of blowing up $1 \leq r \leq 8$ points in general position in $\mathbb{P}^{2}$ is a del Pezzo surface. For $1 \leq r \leq 4$ general points blown up, there is in each case a unique resulting del Pezzo surface. The reason is that for any two sets of points $P_{1}, \ldots, P_{r}$ and $Q_{1}, \ldots, Q_{r}(r \leq 4)$, with each set in general position, there is an element $A \in \operatorname{Aut}\left(\mathbb{P}^{2}\right)=\operatorname{PGL}(3, \mathbb{C})$ with $A\left(P_{i}\right)=Q_{i}(1 \leq i \leq r)$.

For our understanding of del Pezzo surfaces, the following facts about the anticanonical line bundle are also very important.

Facts 2.3 Let $1 \leq r \leq 8$. Let $X$ be obtained by blowing up $r$ general points. Let $E_{i}$ denote the exceptional $(-1)$-curves that are the pre-images of the $r$ points. Let $\pi: X \rightarrow \mathbb{P}^{2}$ denote the blow-up map. Then

$$
K_{X}=\pi^{*} K_{\mathbb{P}^{2}}+\sum_{i=1}^{r} E_{i} .
$$

This yields

$$
\operatorname{dim} H^{0}\left(X,-K_{X}\right)=10-r .
$$

For $1 \leq r \leq 6$, the complete linear system $\left|-K_{X}\right|$ gives an embedding into $\mathbb{P}^{9-r}=\mathbb{P}^{d_{X}}$. For $r=7$, it gives a double cover of $\mathbb{P}^{2}$. The complete linear system $\left|-2 K_{X}\right|$ gives an embedding into $\mathbb{P}^{6}$. For $r=8,\left|-K_{X}\right|$ has a unique base point, $\left|-2 K_{X}\right|$ gives a double cover of a singular quadric surface in $\mathbb{P}^{3}$, and $\left|-3 K_{X}\right|$ gives an embedding into $\mathbb{P}^{6}$.

Finally, it turns out that, on every del Pezzo surface of degree at most 7, the number of $(-1)$-curves exceeds $r$. The reason is that, when blowing up two points in $\mathbb{P}^{2}$, the proper transform of the unique line through the two points becomes a (-1)-curve as well. When blowing up five points, the unique conic through the five points also becomes a $(-1)$-curve. It is easy to count these $(-1)$-curves: for $r=1, \ldots, 8$, their numbers are $1,3,6,10,16,27,56,240$, respectively. Interestingly, for $r=1, \ldots, 6$, under the map given by $\left|-K_{X}\right|$, all (-1)-curves become lines in projective space. Therefore, they are often referred to as lines on $X$.

Remark 2.4 It seems appropriate to remark that most facts about the automorphism groups of del Pezzo surfaces used below were already known in the 19th century (see [34]). The references to more modern treatments given throughout the text are meant for the reader's convenience, not to apportion credit.

\section{Nadel's method of multiplier ideal sheaves}

The following is the standard definition of the multiplier ideal sheaf pertaining to a plurisubharmonic function on a complex manifold. 
Definition-Theorem 3.1 [25] Let $\varphi$ be a plurisubharmonic function on the complex manifold $X$. Then the multiplier ideal sheaf $\mathcal{I}(\varphi)$ is the subsheaf of $\mathcal{O}_{X}$ defined by

$$
\mathcal{I}(\varphi)(U)=\left\{f \in \mathcal{O}_{X}(U):|f|^{2} e^{-\varphi} \in L_{l o c}^{1}(U)\right\}
$$

for every open set $U \subseteq X$. It is a coherent subsheaf.

Multiplier ideal sheaves have turned out to be very useful in algebraic geometry, mainly because of the following vanishing theorem. They are usually defined using the notion of a singular hermitian metric on a line bundle, which in general is a metric $h$ that is given on a small open set $U$ by $h=e^{-\varphi}$, where $\varphi$ is $L^{1}(U)$. If $\varphi$ is plurisubharmonic for every $U$, the multiplier ideal sheaf $\mathcal{I}(h)$ attached to $h$ is defined by $\mathcal{I}(h)(U)=\mathcal{I}(\varphi)(U)$ if $h=e^{-\varphi}$ on $U$.

Theorem 3.2 (Nadel's vanishing theorem) Let X be a compact complex Kähler manifold. Let $L$ be a line bundle on $X$ equipped with a singular hermitian metric such that the curvature current $-\frac{i}{2 \pi} \partial \bar{\partial} \log h$ is positive definite in the sense of currents, i.e., there is a smooth positive definite $(1,1)$-form $\omega$ and $\varepsilon>0$ such that $-\frac{i}{2 \pi} \partial \bar{\partial} \log h \geq \varepsilon \omega$. Then

$$
H^{q}\left(X,\left(K_{X}+L\right) \otimes \mathcal{I}(h)\right)=0 \text { for all } q \geq 1 .
$$

It is well-known that the existence of a Kähler-Einstein metric is equivalent to the solvability of a certain Monge-Ampère equation. If no Kähler-Einstein metric exists, then the continuity method to solve the Monge-Ampère equation must fail. The key idea of [25] is to capture the failure in the form of an invariant non-trivial multiplier ideal sheaf, as expressed in the following theorem (see also [6] for a concise presentation of the details).

Theorem 3.3 (Nadel's existence criterion for Kähler-Einstein metrics) Let X be a Fano manifold, i.e., let $-K_{X}$ be positive. Assume that $X$ does not have a Kähler-Einstein metric. Then, for all compact $G \subseteq \operatorname{Aut}(X)$, the line bundle $-K_{X}$ possesses a $G$-invariant singular hermitian metric $h=h_{0} e^{-\varphi}$, with $h_{0}$ a smooth $G$-invariant metric of smooth positive definite curvature $\omega_{0}$ and $\varphi \in L_{l o c}^{1}(X) G$-invariant, such that

(i) the curvature current $\Theta_{h}$ of $h$ satisfies

$$
\Theta_{h}=-\frac{i}{2 \pi} \partial \bar{\partial} \log h=\omega_{0}+\frac{i}{2 \pi} \partial \bar{\partial} \varphi \geq 0,
$$

(ii) $\forall \gamma \in] \frac{n}{n+1}, 1\left[: 0 \neq \mathcal{I}(\gamma \varphi) \neq \mathcal{O}_{X}\right.$.

The multiplier ideal sheaf $\mathcal{I}(\gamma \varphi)$ is also G-invariant. In particular, every element of $G$ maps the zero-set $V(\mathcal{I}(\gamma \varphi))$ to itself.

Using the Nadel vanishing theorem, Nadel's criterion yields the following corollaries.

Corollary 3.4 Let $X, G, h_{0}, \omega_{0}, \varphi, h, \Theta_{h}, \gamma$ be as in Theorem 3.3 (Nadel's criterion). Then

$$
H^{q}(X, \mathcal{I}(\gamma \varphi))=0 \quad \forall q \geq 1 .
$$

Proof The proof consists of applying Nadel's vanishing theorem with $L=-K_{X}, h_{\gamma}=$ $h_{0} e^{-\gamma \varphi}$. In order to do that, we have to verify that the curvature satisfies the assumption in Nadel's vanishing theorem:

$$
\begin{aligned}
-\frac{i}{2 \pi} \partial \bar{\partial} \log h_{\gamma} & =-\frac{i}{2 \pi} \partial \bar{\partial} \log h_{0} e^{-\gamma \varphi} \\
& =-(1-\gamma) \frac{i}{2 \pi} \partial \bar{\partial} \log h_{0}-\gamma \frac{i}{2 \pi} \partial \bar{\partial} \log \left(h_{0} e^{\varphi}\right) \\
& \geq(1-\gamma) \omega_{0}+\gamma \Theta_{h} \\
& \geq(1-\gamma) \omega_{0} .
\end{aligned}
$$


Now that we have established the required positivity, it can be concluded that

$$
H^{q}\left(X,\left(K_{X}-K_{X}\right) \otimes \mathcal{I}(\gamma \varphi)\right)=H^{q}(X, \mathcal{I}(\gamma \varphi))=0 \quad \forall q \geq 1 .
$$

Corollary 3.5 Let $X, G, h_{0}, \omega_{0}, \varphi, h, \Theta_{h}, \gamma$ be as in Theorem 3.3 (Nadel's criterion). Let $V_{\gamma}=V(\mathcal{I}(\gamma \varphi)) \neq \emptyset$. Then

$$
H^{q}\left(V_{\gamma}, \mathcal{O}_{V_{\gamma}}\right)= \begin{cases}\mathbb{C} & \text { if } q=0, \\ 0 & \text { if } q \geq 1 .\end{cases}
$$

Proof Since $-K_{X}$ is ample, it follows from Kodaira's Vanishing Theorem that

$$
H^{q}\left(X, K_{X}-K_{X}\right)=H^{q}\left(X, \mathcal{O}_{X}\right)=0 \quad \forall q \geq 1 .
$$

Applying this together with Nadel's Vanishing Theorem to the long exact sequence of

$$
0 \rightarrow \mathcal{I}(\gamma \varphi) \rightarrow \mathcal{O}_{X} \rightarrow \mathcal{O}_{V_{\gamma}} \rightarrow 0
$$

yields the result.

Corollary 3.5 tells us that if $\operatorname{dim} V_{\gamma}=0$, then $V_{\gamma}$ is an isolated point that is a fixed point of $G$, i.e., the point constitutes its full orbit under the action of $G$. Moreover, if $\operatorname{dim} V_{\gamma}=1$, it follows that $V_{\gamma}$ is a tree of smooth rational curves (see [25, Theorem 4.4]).

We now explain how the proofs of Theorems 1.2 and 1.3 will be conducted.

First, we consider the case $r \leq 5$.

The key observation in this case is that Theorem 3.3 can be simplified to Criterion 3.7 below, based on the following Theorem [25, Theorem 4.5]. It is proven by an induction argument.

Theorem 3.6 Let $G \subset \operatorname{Aut}(X)$ be a finite subgroup acting without fixed points on $V_{\gamma}$. Then some irreducible component of $V_{\gamma}$ is invariant under $G$. This component is a smooth rational curve.

Criterion 3.7 Let $X$ be a del Pezzo surface. Let there be a finite subgroup $G$ of $\operatorname{Aut}(X)$ which acts on $X$ without fixed points. Assume that $G$ acts effectively on all $G$-invariant curves on $X$. If $G$ is not isomorphic to any one of the following finite groups:

(i) the cyclic group: $\mathbb{Z}_{n}(n \in \mathbb{N})$,

(ii) the dihedral group: $D_{2 n}=\mathbb{Z}_{n} \rtimes \mathbb{Z}_{2}(n \in \mathbb{N})$,

(iii) the alternating group on four letters: $A_{4}$,

(iv) the alternating group on five letters: $A_{5}$,

(v) the symmetric group on four letters: $S_{4}$,

then the statement in the conclusion of Theorem 3.3 is false. In particular, there exists a Kähler-Einstein metric on $X$.

Proof Let us assume that there exists a one-dimensional $G$-invariant zero-set of a multiplier ideal sheaf. Since $G$ acts effectively on all $G$-invariant curves on $X$, in particular it will act effectively on the $G$-invariant smooth rational curve whose existence is established in Theorem 3.6. However, the finite subgroups of $\operatorname{Aut}\left(\mathbb{P}^{1}\right)$ are precisely the ones given in the above list (see [12, Chapter 2]), which yields a contradiction.

Since $G$ acts without fixed points, there also are no zero-dimensional zero-sets of multiplier ideal sheaves. 
Secondly, we consider the case of 6 or more points.

We need the following relative version of Corollary 3.5, whose proof commonly is based on the Leray spectral sequence (see $[21,26])$.

Corollary 3.8 In the situation of Theorem 3.3 (Nadel's criterion), let $\pi: X \rightarrow Y$ be a surjective morphism to a projective manifold. Then $R^{i} \pi_{*}(\mathcal{I}(\gamma \varphi))=0$ for $i \geq 1$.

Remark 3.9 Moreover, by Kodaira's Vanishing Theorem, it is also true that $R^{i} \pi_{*}\left(\mathcal{O}_{X}\right)=0$ $(i \geq 1)$ and therefore finally $R^{i} \pi_{*}\left(\mathcal{O}_{V_{\gamma}}\right)=0(i \geq 1)$. However, we won't be needing these two properties in the cases we consider.

We will also need the following simple criterion for the connectedness of fibers of morphisms.

Definition 3.10 A morphism between topological spaces is called connected if all its fibers are connected.

Proposition 3.11 Let $f: M \rightarrow N$ be a proper morphism of complex manifolds. If the morphism $\mathcal{O}_{N} \rightarrow f_{*} \mathcal{O}_{M}$ is surjective, then $f$ is connected.

Based on Proposition 3.11, we now have

Theorem 3.12 [26, Theorem 3.1] In the situation of Theorem 3.3 (Nadel's criterion), let $f: X \rightarrow Y$ be a connected morphism to a complex projective manifold $Y$. Then $\left(V_{\gamma}\right)_{y}=$ $f^{-1}(y) \cap V_{\gamma}$ is connected.

In Sect. 7, we will see that these statements yield Theorem 1.3.

\section{The case of 3 points blown up}

Let $X$ be a del Pezzo surface obtained from blowing up three points. W.1.o.g., we assume these to be $P_{1}=[1,0,0], P_{2}=[0,1,0], P_{3}=[0,0,1]$. It is remarked in the introduction to [25] that this case can be handled easily based on the fact that $X$ is toric. While this is true, we would like to treat this case in line with the (non-toric) cases $r=4,5$, using a finite group of automorphisms.

First, we determine the full automorphism group of $X$.

Let $H$ be the subgroup of elements of $\operatorname{PGL}(3, \mathbb{C})$ that preserve the set $\left\{P_{1}, P_{2}, P_{3}\right\}$ :

$$
H=\left\{A \in \operatorname{PGL}(3, \mathbb{C}):\left\{A\left(P_{1}\right), A\left(P_{2}\right), A\left(P_{3}\right)\right\}=\left\{P_{1}, P_{2}, P_{3}\right\}\right\} .
$$

The elements of $H$ clearly extend to automorphisms of $X$.

Next, consider the standard quadratic Cremona transformation on $\mathbb{P}^{2}$ :

$$
\operatorname{Cr}\left(\left[X_{0}, X_{1}, X_{2}\right]\right)=\left[X_{1} X_{2}, X_{0} X_{2}, X_{0} X_{1}\right] .
$$

$\mathrm{Cr}$ is a birational involution of $\mathbb{P}^{2}$ that is a morphism outside the set $\left\{P_{1}, P_{2}, P_{3}\right\}$. It lifts to an automorphism of $X$ (see [14, Example V.4.2.3]). We will abuse notation and denote the induced automorphism of $X$ again by $\mathrm{Cr}$.

It is a well-known fact (see, e.g., [20]) that

$$
\operatorname{Aut}(X)=H \rtimes\{1, \mathrm{Cr}\}=H \rtimes \mathbb{Z}_{2} .
$$


This identity can also be written as

$$
\operatorname{Aut}(X)=\left(\mathbb{C}^{*} \times \mathbb{C}^{*}\right) \rtimes\left(S_{3} \times \mathbb{Z}_{2}\right),
$$

where $S_{3} \subset \operatorname{PGL}(3, \mathbb{C})$ is the subgroup of projectivities that act on $\mathbb{P}^{2}$ by permuting the coordinates, i.e., for $\sigma \in S_{3}$,

$$
\sigma\left(\left[X_{0}, X_{1}, X_{2}\right]\right)=\left[X_{\sigma(0)}, X_{\sigma(1)}, X_{\sigma(2)}\right] .
$$

Moreover, $\mathbb{C}^{*} \times \mathbb{C}^{*}$ acts by multiplicitation on, say, the last two coordinates.

In order to obtain a finite subgroup of $\operatorname{Aut}(X)$ to use in Criterion 3.7, we note that the Klein four-group $\mathbb{Z}_{2} \times \mathbb{Z}_{2} \subset \mathbb{C}^{*} \times \mathbb{C}^{*}$ acts on $X$ as follows:

$$
(\alpha, \beta)\left(\left[X_{0}, X_{1}, X_{2}\right]\right)=\left[X_{0},(-1)^{\alpha} X_{1},(-1)^{\beta} X_{2}\right] .
$$

Let

$$
G=\left(\mathbb{Z}_{2} \times \mathbb{Z}_{2}\right) \rtimes\left(S_{3} \times \mathbb{Z}_{2}\right)
$$

Lemma 4.1 The action of $G$ is fixed point free.

Proof The only fixed point of the action of $S_{3}$ is $[1,1,1]$. However, this point is not fixed under the action of $(0,1) \in \mathbb{Z}_{2} \times \mathbb{Z}_{2}$.

Remark 4.2 It is well-known that $\left(\mathbb{Z}_{2} \times \mathbb{Z}_{2}\right) \rtimes S_{3}=S_{4}$. From a group theoretic point of view, the statement of Lemma 4.1 is implied by the fact that $S_{4}$ admits no complex two-dimensional faithful representations (on the tangent space of a point of $X$ ). The non-existence of such representations is a well-known fact in the representation theory of finite groups (see [8, p. 148]).

Lemma 4.3 The group $G$ acts effectively on any $G$-invariant irreducible curve.

Proof The elements of $G$ are induced by maps of $\mathbb{P}^{2}$ which are either of the form

$$
\left[X_{0}, X_{1}, X_{2}\right] \mapsto\left[X_{\sigma(0)},(-1)^{\alpha} X_{\sigma(1)},(-1)^{\beta} X_{\sigma(2)}\right]
$$

or

$$
\left[X_{0}, X_{1}, X_{2}\right] \mapsto\left[X_{\sigma(1)} X_{\sigma(2)},(-1)^{\alpha} X_{\sigma(0)} X_{\sigma(2)},(-1)^{\beta} X_{\sigma(0)} X_{\sigma(1)}\right] .
$$

Now let us assume the existence of a $G$-invariant irreducible curve $C$ on $X$ which is fixed point-wise by a non-trivial element $g \in G$. We shall derive a contradiction.

First, we note that none of the six $(-1)$-curves are $G$-invariant. Thus, they are different from $C$, and $C$ has to be the proper transform of a curve $D$ in $\mathbb{P}^{2}$.

The first case is that $g$ is induced by a map $\hat{g}$ of the form (1). The map $\hat{g}$ has to fix $D$ point-wise. However, $\hat{g}$ is linear, thus $D$ has to be a line. This is because, if the degree of $D$ were greater than one, then any secant line of $D$ would be preserved, which would mean in turn that the induced mapping on the dual $\mathbb{P}^{2 *}$ is the identity. Thus, $\hat{g}$ itself would have to be the identity.

Because of the $S_{3}$-invariance, the only possibility for $D$ is the line $X_{0}+X_{1}+X_{2}=0$. However, this line is not invariant under

$$
\left[X_{0}, X_{1}, X_{2}\right] \mapsto\left[X_{0},-X_{1}, X_{2}\right]
$$

Contradiction. 
The second case is that $g$ is induced by a map $\hat{g}$ of the form (2). Again, the map $\hat{g}$ has to fix $D$ point-wise. For symmetry reasons, we can confine ourselves w.l.o.g. to $\sigma=(01)$ or $\sigma=(012)$. Here, we are using the usual cycle notation for permutations.

Let us first take $\sigma=(01)$ and find the fixed point set of $\hat{g}$. The equation

$$
\left[X_{0} X_{2},(-1)^{\alpha} X_{1} X_{2},(-1)^{\beta} X_{0} X_{1}\right]=\left[X_{0}, X_{1}, X_{2}\right]
$$

is equivalent to the existence of $\lambda \in \mathbb{C}^{*}$ such that

$$
X_{0} X_{2}=\lambda X_{0}, \quad(-1)^{\alpha} X_{1} X_{2}=\lambda X_{1}, \quad(-1)^{\beta} X_{0} X_{1}=\lambda X_{2} .
$$

If one of the $X_{i}$ were equal to zero, then clearly the remaining two would have to be zero as well in order to satisfy the above system of equations. Thus, we can assume $X_{i} \neq 0$ for $i=0,1,2$. In this case, the system reduces to

$$
(-1)^{\alpha} X_{1}=X_{1}, \quad(-1)^{\beta} X_{0} X_{1}=X_{2}^{2} .
$$

Obviously, $(-1)^{\alpha}$ has to equal one, and the system reduces to the equation $X_{2}^{2}-(-1)^{\beta} X_{0} X_{1}=$ 0 for $D$. However, regardless of the sign, this equation is not preserved by the action of the transposition (12) on $\mathbb{P}^{2}$. Contradiction.

Next, let $\sigma=(012)$. The equation

$$
\left[X_{0} X_{2},(-1)^{\alpha} X_{0} X_{1},(-1)^{\beta} X_{1} X_{2}\right]=\left[X_{0}, X_{1}, X_{2}\right]
$$

is equivalent to the existence of $\lambda \in \mathbb{C}^{*}$ such that

$$
X_{0} X_{2}=\lambda X_{0}, \quad(-1)^{\alpha} X_{0} X_{1}=\lambda X_{1}, \quad(-1)^{\beta} X_{1} X_{2}=\lambda X_{2} .
$$

This system reduces to

$$
(-1)^{\alpha} X_{0}=X_{2}, \quad(-1)^{\beta} X_{1}=X_{2},
$$

which defines the point $\left[1,(-1)^{\alpha-\beta},(-1)^{\alpha}\right]$, and not a curve. Contradiction.

We finally observe that $G$ is a group of order 48 which is clearly not isomorphic to any of the groups listed in Criterion 3.7 as finite subgroups of Aut $\left(\mathbb{P}^{1}\right)$. Thus, Lemmas 4.1 and 4.3 allow us to apply Criterion 3.7, and we have established Main Theorem 1.2 in the case of three points blown up.

Remark 4.4 Note that the use of the automorphism induced by the quadratic Cremona transformation is crucial. The group $\left(\mathbb{Z}_{2} \times \mathbb{Z}_{2}\right) \rtimes S_{3}=S_{4}$ is a subgroup of $\operatorname{Aut}\left(\mathbb{P}^{1}\right)$, and it cannot be used in applying Criterion 3.7.

\section{The case of 4 points blown up}

Let $X$ be a del Pezzo surface obtained from blowing up four points. We may, and do, assume these to be $P_{1}=[1,0,0], P_{2}=[0,1,0], P_{3}=[0,0,1], P_{4}=[1,1,1]$. It is known that $\operatorname{Aut}(X)$ is the Weyl group of the root system of Dynkin type $D_{5}$, which is $S_{5}$ (see [20]). However, we would like to understand $\operatorname{Aut}(X)$ more concretely.

First all of, there is a subgroup $S_{4}$ of projectivities in $\operatorname{PGL}(3, \mathbb{C})=\operatorname{Aut}\left(\mathbb{P}^{2}\right)$ that preserve the set $\left\{P_{1}, P_{2}, P_{3}, P_{4}\right\}$. These projectivities lift to $X$, and we can write $S_{4} \subset \operatorname{Aut}(X)$.

In addition, there exists for every $i=1, \ldots, 4$ a quadratic Cremona transformation $\mathrm{Cr}_{i}$ that leaves $P_{i}$ fixed and has the three remaining points as indeterminacy locus. Note that such a $\mathrm{Cr}_{i}$ is only defined up to the action of the $S_{3} \subset S_{4}$ consisting of automorphisms fixing $P_{i}$. 
For our purposes, it does not matter which $\mathrm{Cr}_{i}$ we choose. All $\mathrm{Cr}_{i}$ extend to automorphisms of $X$. In light of this, we can write $\operatorname{Aut}(X)$ set-theoretically as a disjoint union

$$
\operatorname{Aut}(X)=S_{4} \uplus\left(\biguplus_{i=1}^{4} \mathrm{Cr}_{i} \circ S_{4}\right) \text {. }
$$

Lemma 5.1 The action of $\operatorname{Aut}(X)$ is fixed point free.

Proof The element of $\operatorname{Aut}(X)$ induced by

$$
\left(\begin{array}{ccc}
0 & 0 & 1 \\
-1 & 0 & 1 \\
0 & -1 & 1
\end{array}\right)
$$

has precisely three fixed points on $X$, namely those points on $X$ lying over

$$
[1,0,1],[1,1+i, i],[1,1-i,-i]
$$

in $\mathbb{P}^{2}$. On the other hand, the fixed point set of the element of $\operatorname{Aut}(X)$ induced by $\mathrm{Cr}_{4}$ consists of the three points lying over

$$
[-1,1,1],[1,1,-1],[1,-1,1]
$$

and the $(-1)$-curve over $[1,1,1]$. Since there is no point appearing in both fixed point sets, the Lemma is proven.

Remark 5.2 We already noted that $S_{4}$ admits no complex two-dimensional faithful representations. Since representations clearly remain faithful under restriction to subgroups, $S_{5}$ does not admit any such representations either. So Lemma 5.1 follows directly from representation theory, without any explicit computations.

Lemma 5.3 Aut $(X)$ acts effectively on any $\operatorname{Aut}(X)$-invariant irreducible curve.

Proof For any given element of $\operatorname{Aut}(X)$, it is easy to list the irreducible curves that are left point-wise fixed by the given element (if any exist). However, none of these curves are $\operatorname{Aut}(X)$-invariant. The precise arguments are completely analogous to those in the proof of Lemma 4.3.

Lemmas 5.1 and 5.3 allow us to apply Criterion 3.7 to establish Main Theorem 1.2 in the case of four points blown up.

\section{The case of 5 points blown up}

Let $X$ be a del Pezzo surface obtained by blowing up five points. We can find an automorphism of $\mathbb{P}^{2}$ that takes the five points to $P_{1}=[1,0,0], P_{2}=[0,1,0], P_{3}=[0,0,1]$, $P_{4}=[1,1,1], P_{5}=[a, b, c]$, with $(a, b, c) \in\left(\mathbb{C}^{*} \times \mathbb{C}^{*} \times \mathbb{C}^{*}\right) \backslash\{(1,1,1)\}$. (The reason for $a, b, c \neq 0$ is that no three of these points lie on a line).

The structure of $\operatorname{Aut}(X)$ is worked out in [17]. It turns out that it is always of the form

$$
\operatorname{Aut}(X)=\mathbb{Z}_{2}^{4} \rtimes G_{P_{5}},
$$

where $G_{P_{5}}$ is a subgroup of $S_{5}$. The possibilities for $G_{P_{5}}$ are 
(i) $\{$ id $\}$,

(ii) $\mathbb{Z}_{2}$,

(iii) $\mathbb{Z}_{4}$,

(iv) $\mathbb{Z}_{3} \rtimes \mathbb{Z}_{2}$,

(v) $\mathbb{Z}_{5} \rtimes \mathbb{Z}_{2}$.

The elements of $G_{P_{5}}$ are lifts of those elements of PGL(3, $\left.\mathbb{C}\right)$ that preserve the set $\left\{P_{1}, P_{2}\right.$, $\left.P_{3}, P_{4}, P_{5}\right\}$. For a generic point $P_{5}$, we have $G_{P_{5}}=\{\mathrm{id}\}$.

For our purposes, it suffices to argue with elements of $\mathbb{Z}_{2}^{4} \subseteq \operatorname{Aut}(X)$, regardless of the nature of $G_{P_{5}}$.

Lemma 6.1 The action of any subgroup of $\operatorname{Aut}(X)$ isomorphic to $\mathbb{Z}_{2}^{3}$ or $\mathbb{Z}_{2}^{4}$ is fixed point free.

Proof Instead of using explicit computations, we will argue purely with methods from representation theory. Let $H$ be a subgroup of $\operatorname{Aut}(X)$ isomorphic to either $\mathbb{Z}_{2}^{3}$ or $\mathbb{Z}_{2}^{4}$. If $H$ has a fixed point, then it has a faithful two-dimensional complex representation on the tangent space of the fixed point.

Since $H$ is abelian, by Schur's Lemma, the only irreducible representations of $H$ are maps $\rho: H \rightarrow \mathbb{C}^{*}$ (see [9, p. 8]). Since every non-trivial element of $H$ has order two, the image of $\rho$ is either $\{1\}$ or $\{1,-1\}$. Therefore, the image of $H$ in $\operatorname{GL}(2, \mathbb{C})$ is isomorphic to either $\{1\}, \mathbb{Z}_{2}$, or $\mathbb{Z}_{2}^{2}$. We have obtained a contradiction.

Next, let us have a closer look at the elements of $\mathbb{Z}_{2}^{4} \subseteq \operatorname{Aut}(X)$. The following two birational involutions of $\mathbb{P}^{2}$ lift to elements of $\operatorname{Aut}(X)$.

We define $\mathrm{Cr}_{45}$ to be

$$
\mathrm{Cr}_{45}\left(\left[X_{0}, X_{1}, X_{2}\right]\right)=\left[a X_{1} X_{2}, b X_{0} X_{2}, c X_{0} X_{1}\right] .
$$

This is a quadratic Cremona transformation that exchanges $P_{4}$ and $P_{5}$ and has $\left\{P_{1}, P_{2}, P_{3}\right\}$ as indeterminacy locus.

Moreover, we let $\sigma_{1}$ be the following cubic involution.

$$
\begin{aligned}
\sigma_{1}\left(\left[X_{0}, X_{1}, X_{2}\right]\right)= & {\left[-a X_{1} X_{2}\left((c-b) X_{0}+(a-c) X_{1}+(b-a) X_{2}\right),\right.} \\
& X_{1}\left(a(c-b) X_{1} X_{2}+b(a-c) X_{0} X_{2}+c(b-a) X_{0} X_{1}\right), \\
& \left.X_{2}\left(a(c-b) X_{1} X_{2}+b(a-c) X_{0} X_{2}+c(b-a) X_{0} X_{1}\right)\right] .
\end{aligned}
$$

The explicit description of $\sigma_{1}$ tells us that the analog of Lemmas 4.3 and 5.3 is not true in the case of five points blown up. Namely, it is easy to see that the strict transform of the cubic curve $C$ given by

$b(a-c) X_{0}^{2} X_{2}+c(b-a) X_{0}^{2} X_{1}+a(a-c) X_{1}^{2} X_{2}+a(b-a) X_{1} X_{2}^{2}+2 a(c-b) X_{0} X_{1} X_{2}=0$

is precisely the set of points fixed point-wise by the lift of the above $\sigma_{1}$. In addition, the following Lemma tells us that $C$ is invariant under every element of $\mathbb{Z}_{2}^{4}$.

Lemma 6.2 Let $A$ be an abelian group acting on a set $M$. For $g \in A$, let

$$
M^{g}=\{x \in M: g x=x\} .
$$

Then $M^{g}$ is invariant under every element of $A$.

Proof For $x \in M^{g}$, we have

$$
g(h x)=h(g x)=h x .
$$


However, there are several reasons why this observation does not impede our proof.

Closer inspection $[17,20]$ shows that $\mathbb{Z}_{2}^{4} \subseteq \operatorname{Aut}(X)$ contains the lifts of ten quadratic Cremona involutions $\mathrm{Cr}_{i j}(1 \leq i<j \leq 5)$ that exchange $P_{i}, P_{j}$ and have the remaining three points as indeterminacy locus. Again, we abuse notation and denote the maps before and after the lift by the same symbols $\mathrm{Cr}_{i j}$.

Moreover, $\mathbb{Z}_{2}^{4} \subseteq \operatorname{Aut}(X)$ contains the lifts of five cubic involutions $\sigma_{i}(1 \leq i \leq 5)$. By comparing the respective action on the set of the $16(-1)$-curves (which determines any automorphism uniquely, see [20] or [17]), it is easily verified that

$$
\begin{aligned}
\mathrm{Cr}_{i j} \circ \mathrm{Cr}_{k l} & =\mathrm{Cr}_{k l} \circ \mathrm{Cr}_{i j}, \\
\mathrm{Cr}_{i j} \circ \mathrm{Cr}_{j k} & =\mathrm{Cr}_{i k} .
\end{aligned}
$$

Moreover, for $i, j, k, l, m$ all distinct, we have

$$
\sigma_{m}=\mathrm{Cr}_{i j} \circ \mathrm{Cr}_{k l} .
$$

In particular, we have

$$
\sigma_{j}=\mathrm{Cr}_{1 j} \circ \sigma_{1} \quad(2 \leq j \leq 5) .
$$

The $\mathrm{Cr}_{i j}$ have precisely four fixed points each (both before and after the lifting). Thus, the only $\mathbb{Z}_{2}^{4}$-invariant curves on which $\mathbb{Z}_{2}^{4}$ does not act effectively are the lifts of the curves $C$ pertaining to the cubic involutions. However, it is easy to see that for all values $a, b, c \neq 0$, the curves $C$ are smooth elliptic curves. Therefore, there exist no smooth rational curves on $X$ on which $G$ acts ineffectively. We omit the easy details of the above argument and instead argue without assuming any knowledge regarding fixed loci of the $\sigma_{i}$, merely using the group structure of $\mathbb{Z}_{2}^{4}$.

Lemma 6.3 The group $\mathbb{Z}_{2}^{3}$ acts effectively on any $\mathbb{Z}_{2}^{4}$-invariant irreducible curve.

Proof Let $C$ be a $\mathbb{Z}_{2}^{4}$-invariant irreducible curve. Assume that $g \neq$ id acts trivially on $C$. Then $g=\sigma_{i}$ for some $1 \leq i \leq 5$, because the Cremona maps only have four fixed points each. W.l.o.g., let $i=1$.

Because of (4), none of the $\sigma_{j}(2 \leq j \leq 5)$ act trivially on $C$. Thus the group

$$
\mathbb{Z}_{2}^{4} /\left\{\mathrm{id}, \sigma_{1}\right\}=\mathbb{Z}_{2}^{3}
$$

acts effectively on $C$.

Lemmas 6.1 and 6.3 allow us to apply Criterion 3.7 to establish Main Theorem 1.2 in the last remaining case of five points blown up. This completes the proof of Main Theorem 1.2.

\section{The cases of 6,7, or 8 points}

The following is a concise presentation of [26, Section 4.1]. We have added the missing details for the unspecified "careful choice" in the proof of [26, Proposition 4.1].

Lemma 7.1 Let $E_{1}, \ldots, E_{r}$ be any set of $r$ pairwise disjoint (-1)-curves on a del Pezzo surface $X$ of degree $9-r$. Then blowing down $E_{1}, \ldots, E_{r}$ gives a birational morphism $X \rightarrow \mathbb{P}^{2}$.

Proof The image is a del Pezzo surface of degree 9. According to the classification, the only such del Pezzo surface is $\mathbb{P}^{2}$. 
Lemma 7.2 Let $X$ be a del Pezzo surface of degree $d \leq 4$. Let $C$ be any irreducible curve on $X$. Then there are mutually disjoint $(-1)$-curves $\tilde{E}_{1}, \ldots, \tilde{E}_{r}(r=9-d)$ whose blowing down gives $\tilde{\pi}: X \rightarrow \mathbb{P}^{2}$ with $\operatorname{dim} \tilde{\pi}(C)=1$ and $\operatorname{deg} \tilde{\pi}(C) \geq 2$.

Proof Let $E_{1}, \ldots, E_{r}$ be the original exceptional curves for the blowing up $\pi: X \rightarrow \mathbb{P}^{2}$. If $C$ happens to be one of them, say $C=E_{1}$, then we can contract $E_{12}, E_{13}, E_{23}$ instead of $E_{1}=C, E_{2}, E_{3}$, where $E_{i j}$ is the strict transform of the line through $P_{i}$ and $P_{j}$. Therefore, we can assume w.l.o.g. that $C$ does not get contracted by $\pi$, i.e., $\operatorname{dim} \pi(C)=1$.

If $\pi(C)$ is a line in $\mathbb{P}^{2}$ (we are done otherwise), then under any blowing down map, at most two image points of exceptional curves can lie on $\pi(C)$, because the image points will be in general position. In any case, we can assume w.l.o.g. that the image points of $E_{3}, E_{4}, E_{5}$ are each not on $\pi(C)$ (here we use $r \geq 5$ ). Now let

$$
\tilde{E}_{1}=E_{1}, \tilde{E}_{2}=E_{2}, \tilde{E}_{3}=E_{34}, \tilde{E}_{4}=E_{35}, \tilde{E}_{5}=E_{45} .
$$

If $r \geq 6$, also let

$$
\tilde{E}_{6}=E_{6}, \ldots, \tilde{E}_{r}=E_{r} .
$$

We have

$$
\tilde{\pi}\left(E_{34}\right), \tilde{\pi}\left(E_{35}\right), \tilde{\pi}\left(E_{45}\right) \in \tilde{\pi}(C) .
$$

This implies deg $\tilde{\pi}(C) \geq 2$, because $\tilde{\pi}\left(E_{34}\right), \tilde{\pi}\left(E_{35}\right), \tilde{\pi}\left(E_{45}\right)$ are three points in general position.

Proposition 7.3 Let $X$ be a del Pezzo surface of degree $d \leq 3$. Then $-K_{X}$ does not carry a singular hermitian metric has in Theorem 3.3 (Nadel's criterion) such that $\operatorname{dim} V(\mathcal{I}(h))=1$.

Proof We argue by contradiction. W.l.o.g, let $C=V(\mathcal{I}(h))$ be an irreducible curve. Let $\pi: X \rightarrow \mathbb{P}^{2}$ be such that $\operatorname{deg} \pi(C) \geq 2$ ( $\pi$ exists by Lemma 7.2). For $1 \leq i \leq r=9-d$, let $\pi\left(E_{i}\right)=P_{i}$.

Case 1: $\operatorname{deg} \pi(C)=2$. Since $r \geq 6$, there is $1 \leq i \leq r$ such that $P_{i} \notin \pi(C)$. W.1.o.g., let $i=r$. Note that $\pi$ factors as

$$
X \stackrel{\pi_{1, \ldots, r-1}}{\longrightarrow} \tilde{\mathbb{P}}^{2} \stackrel{\pi_{r}}{\longrightarrow} \mathbb{P}^{2},
$$

where $\pi_{r}$ denotes the blow up of the point $P_{r}$, and $\pi_{1, \ldots, r-1}$ denotes the blow up of the points $\pi_{r}^{-1}\left(P_{1}\right), \ldots, \pi_{r}^{-1}\left(P_{r-1}\right)$. The key fact is that a general line through $P_{r}$ intersects $\pi(C)$ (precisely) twice in $\mathbb{P}^{2} \backslash\left\{P_{r}\right\}$. Let $\tau$ denote the fibration $\tilde{\mathbb{P}}^{2} \rightarrow \mathbb{P}^{1}$ whose fibers are lines through $P_{r}$. Let $f$ denote the composition

$$
X \stackrel{\pi_{1, \ldots, r-1}}{\longrightarrow} \tilde{\mathbb{P}}^{2} \stackrel{\tau}{\rightarrow} \mathbb{P}^{1}
$$

Note that the sets $C_{y}=f^{-1}(y) \cap C$ have cardinality at least two for general $y \in \mathbb{P}^{1}$, which is impossible by Theorem 3.12.

Case 2: $\operatorname{deg} \pi(C) \geq 3$. If there exists an index $1 \leq i \leq r$ such that $P_{i} \notin \pi(C)$, then we can argue exactly as we did in the previous case. So let us assume that for all $1 \leq i \leq r$, $P_{i} \in \pi(C)$.

In order to argue again with the connectedness of fibers, we need to find $i_{0}$ such that a general line through $P_{i_{0}}$ intersects $\pi(C)$ in at least two additional distinct points. Let $d=\operatorname{deg} \pi(C)$. If mult $P_{i_{0}} \pi(C) \leq d-2$, then the $i_{0}$ is obviously of the kind we are looking 
for. We claim that such an $i_{0}$ always exists. To argue by contradiction, assume that for all $1 \leq i \leq r$, we have mult $P_{i_{0}} \pi(C) \geq d-1$.

Choose a smooth cubic curve $F$ through $P_{1}, \ldots, P_{r}$. Then the intersection number of $F$ and $\pi(C)$ is

$$
F \cdot \pi(C)=3 d
$$

It is a basic fact from intersection theory that

$$
3 d=F \cdot \pi(C) \geq \sum_{x \in F \cap \pi(C)} \operatorname{mult}_{x} F \cdot \operatorname{mult}_{x} \pi(C) \geq \sum_{x \in F \cap \pi(C)} \operatorname{mult}_{x} \pi(C) \geq r(d-1) .
$$

Consequently, $r \geq(r-3) d$. It is easy to check that for $r \geq 6$, this implies $d \leq 2$, a contradiction.

Theorem 1.3 [26] Let $X$ be a del Pezzo surface of degree no more than three. If Aut $(X)$ acts without fixed points on $X$, then $X$ has a Kähler Einstein metric.

Proof Since there are no zero- or one-dimensional zero-sets of Aut $(X)$-invariant multiplier ideal sheaves, the theorem follows from Theorem 3.3 (Nadel's criterion).

To clarify the extent to which Theorem 1.3 can actually be applied to prove the existence of Kähler-Einstein metrics on del Pezzo surfaces of degree no more than three, we make the following remarks. An excellent reference with a lucid exposition is [7, Sect. 10].

Let $X$ be a del Pezzo surface of degree one. The unique base point of the linear system $\left|-K_{X}\right|$ is fixed by all automorphisms. Therefore, unfortunately, $\operatorname{Aut}(X)$ acts with a fixed point regardless of the nature of $X$.

On a del Pezzo surface $X$ of degree two, the linear system $\left|-K_{X}\right|$ gives a two-sheeted cover of $\mathbb{P}^{2}$ branched along a smooth curve $C$ of degree 4 in $\mathbb{P}^{2}$. This cover defines an involutive automorphism of $X$ called the Geiser involution, which lies in the center of $\operatorname{Aut}(X)$. The corresponding quotient group $G^{\prime}=\operatorname{Aut}(X) / \mathbb{Z}_{2}$ acts effectively on $C$. If the action of $\operatorname{Aut}(X)$ on $X$ has a fixed point, then so does the action of $G^{\prime}$ on $C$. At the fixed point, $G^{\prime}$ has a faithful representation on the one-dimensional tangent space to $C$, so $G^{\prime}$ must be a cyclic group $\mathbb{Z}_{n}$. Therefore, the action of $\operatorname{Aut}(X)$ has a fixed point only if $\operatorname{Aut}(X)$ is a cyclic central extension of $\mathbb{Z}_{n}$ by $\mathbb{Z}_{2}$. For a list of surfaces $X$ for which this is not the case, see [7, Table 10.4].

For a generic del Pezzo surface $X$ of degree three, $\operatorname{Aut}(X)$ is the trivial group (see [20]). However, since del Pezzo surfaces of degree 3 are precisely the smooth cubic hypersurfaces of $\mathbb{P}^{3}$, there are many non-generic $X$ which have extra automorphisms. The following paragraphs give a sufficient criterion for the action of $\operatorname{Aut}(X)$ to be fixed point free.

Assume that $x \in X$ is a fixed point of the action of $\operatorname{Aut}(X)$. Then the canonical map $\operatorname{GL}(2, \mathbb{C}) \rightarrow \operatorname{PGL}(2, \mathbb{C})$ induces a map $\operatorname{Aut}(X) \rightarrow G^{\prime}=\operatorname{Aut}(X) / Z(\operatorname{Aut}(X))$, where $Z(\operatorname{Aut}(X))$ denotes the center. The tangent plane to $X$ at $x$ intersects $X$ in a singular cubic plane curve $C$, and there are one, two or three lines through $x$ in the tangent plane that are tangent to $C$ at $x$. Let $T \subset \mathbb{P}^{1}$ be the set of points corresponding to these lines in the projectivized tangent plane at $x$. Note that $T$ is invariant under the action of $G^{\prime}$ on $\mathbb{P}^{1}$.

If $T=\left\{p_{1}\right\}$, then it follows from the classification of finite subgroups of $\operatorname{PGL}(2, \mathbb{C})$ mentioned in Sect. 3 that $G^{\prime}$ is a cyclic group $\mathbb{Z}_{n}$.

If $T=\left\{p_{1}, p_{2}\right\}$, then the classification yields that either $G^{\prime}$ is a cyclic group $\mathbb{Z}_{n}$ or a dihedral group $\mathbb{Z}_{n} \rtimes \mathbb{Z}_{2}$.

If $T=\left\{p_{1}, p_{2}, p_{3}\right\}$, then $G^{\prime} \subset S_{3}$, i.e., one of the following holds: $G^{\prime}=\{\mathrm{id}\}, G^{\prime}=\mathbb{Z}_{2}$, $G^{\prime}=\mathbb{Z}_{3}$, or $G^{\prime}=\mathbb{Z}_{3} \rtimes \mathbb{Z}_{2}=S_{3}$. 
We have just shown that if $\operatorname{Aut}(X)$ acts with a fixed point, then it is a central extension of one the above $G^{\prime}$ by a finite cyclic group. Pairing this knowledge with [7, Table 10.3], it is a simple task to determine which automorphism groups act without fixed points and therefore carry a Kähler-Einstein metric based on Theorem 1.3. Most notably, the list contains the two examples given by

$$
Z_{0}^{3}+Z_{1}^{3}+Z_{2}^{3}+Z_{3}^{3}=0
$$

and

$$
Z_{0}^{2} Z_{1}+Z_{2}^{2} Z_{0}+Z_{3}^{2} Z_{2}+Z_{1}^{2} Z_{3}=0
$$

The automorphism groups are $\mathbb{Z}_{3}^{3} \rtimes S_{4}$ resp. $S_{5}$. In both cases, the action clearly is fixed point free.

\section{The cases of 1 or 2 points}

Let $X$ be the del Pezzo surface obtained by blowing up one point, which we can assume to be $P=[1,0,0]$. The automorphism group of $X$ is isomorphic to the subgroup of elements of PGL $(3, \mathbb{C})$ that fix $P$.

There is a subgroup isomorphic to $S^{1} \times S^{1}$ of $\operatorname{PGL}(3, \mathbb{C})$ given by

$$
\left(e^{i \alpha}, e^{i \beta}\right)\left(\left[X_{0}, X_{1}, X_{2}\right]\right)=\left[X_{0}, e^{i \alpha} X_{1}, e^{i \beta} X_{2}\right] .
$$

The map

$$
\left[X_{0}, X_{1}, X_{2}\right] \mapsto\left[X_{0}, X_{2}, X_{1}\right]
$$

is also in PGL(3, $\mathbb{C})$, and all of the above maps fix $P$. Therefore, they induce elements of $\operatorname{Aut}(X)$. The subgroup $G$ of $\operatorname{Aut}(X)$ which they generate is a semi-direct product $\left(S^{1} \times\right.$ $\left.S^{1}\right) \rtimes \mathbb{Z}_{2}$.

On $X$ there are two irreducible subvarieties which are each invariant under $G$, namely the exceptional curve and the proper transform of the line $X_{0}=0$. It was remarked in the Introduction that the non-vanishing of Futaki's invariant implies that there cannot be a Kähler-Einstein metric on $X$. It is not a priori clear which of the two curves is the zero-set of the multiplier ideal sheaf arising from the failure of the continuity method. In [27], Nadel gave a necessary condition for a subvariety to arise as such a zero-set, but it is an easy exercise to see that both curves satisfy this criterion. However, in a recent result [10, Theorem 1.2], Futaki and Sano extended Nadel's necessary condition and managed to show that it is the exceptional curve that arises.

Now let $X$ be the del Pezzo surface obtained by blowing up two points, which we can assume to be $P_{1}=[1,0,0]$ and $P_{2}=[0,1,0]$. The automorphism group of $X$ is isomorphic to the subgroup of elements of $\operatorname{PGL}(3, \mathbb{C})$ that preserve the set $\left\{P_{1}, P_{2}\right\}$. The same maps as above induce a subgroup $\left(S^{1} \times S^{1}\right) \rtimes \mathbb{Z}_{2}$ of $\operatorname{Aut}(X)$.

At the moment, it seems to be unknown which of the obvious candidates is the zero-set of the multiplier ideal sheaf arising from the failure of the continuity method. In the first two versions of [10] it was claimed that it is the proper transform of the line through $P_{1}, P_{2}$ that arises. However, this claim has been retracted in the third version. We refer the reader to [10] for more details.

Acknowledgments The author would like to thank I. Dolgachev for helpful comments concerning the automorphism groups of del Pezzo surfaces of degree at most three. 
Open Access This article is distributed under the terms of the Creative Commons Attribution Noncommercial License which permits any noncommercial use, distribution, and reproduction in any medium, provided the original author(s) and source are credited.

\section{References}

1. Aubin, T.: Équations du type Monge-Ampère sur les variétés kähleriennes compactes. C. R. Acad. Sci. Paris Sér. A-B 283(3), Aiii, A119-A121 (1976)

2. Aubin, T.: Équations du type Monge-Ampère sur les variétés kählériennes compactes. Bull. Sci. Math. (2) 102(1), 63-95 (1978)

3. Aubin, T.: Nonlinear analysis on manifolds. Monge-Ampère equations. Grundlehren der Mathematischen Wissenschaften [Fundamental Principles of Mathematical Sciences], vol. 252. Springer, New York (1982)

4. Bourguignon, J.P.: Métriques d'Einstein-Kähler sur les variétés de Fano: obstructions et existence (d'après Y. Matsushima, A. Futaki, S. T. Yau, A. Nadel et G. Tian). Astérisque, (245): Exp. No. 830, 5, 277-305, 1997. Séminaire Bourbaki, vol. 1996/97

5. Demazure, M.: Surfaces de Del Pezzo, I-V. Lecture Notes in Mathematics, vol. 777, pp. 21-61. Springer, Berlin (1980)

6. Demailly, J.-P., Kollár, J.: Semi-continuity of complex singularity exponents and Kähler-Einstein metrics on Fano orbifolds. Ann. Sci. École Norm. Sup. (4) 34(4), 525-556 (2001)

7. Dolgachev, I.V.: Topics in classical algebraic geometry. Lecture notes. http://www.math.lsa.umich.edu/ idolga/lecturenotes.html (2007)

8. Dornhoff, L.: Group representation theory. Part A: Ordinary representation theory. Pure and Applied Mathematics, vol. 7. Marcel Dekker, New York (1971)

9. Fulton, W., Harris, J.: Representation theory. Graduate Texts in Mathematics, vol. 129. Springer, New York (1991) A first course, Readings in Mathematics

10. Futaki, A., Sano, Y.: Multiplier ideal sheaves and integral invariants on toric Fano manifolds. arXiv: math/0711.0614v3 (2008)

11. Futaki, A.: An obstruction to the existence of Einstein Kähler metrics. Invent. Math. 73(3), 437-443 (1983)

12. Grove, L.C., Benson, C.T.: Finite reflection groups. Graduate Texts in Mathematics, vol. 99, 2nd edn. Springer, New York (1985)

13. Griffiths, P., Harris, J.: Principles of algebraic geometry. Wiley Classics Library. Wiley, New York (1994). Reprint of the 1978 original

14. Hartshorne, R.: Algebraic Geometry. Graduate Texts in Mathematics, vol. 52. Springer, New York (1977)

15. Harris, J.: Algebraic Geometry. Graduate Texts in Mathematics, vol. 133. Springer, New York (1995). A first course, Corrected reprint of the 1992 original

16. Heier, G.: Convergence of the Kähler-Ricci flow and multiplier ideal sheaves on del Pezzo surfaces. arXiv:math/0710.5725 (2007). Mich. Math. J. (to appear)

17. Hosoh, T.: Automorphism groups of quartic del Pezzo surfaces. J. Algebra 185(2), 374-389 (1996)

18. Hosoh, T.: Automorphism groups of cubic surfaces. J. Algebra 192(2), 651-677 (1997)

19. Kohn, J.J.: Subellipticity of the $\bar{\partial}$-Neumann problem on pseudo-convex domains: sufficient conditions. Acta Math. 142(1-2), 79-122 (1979)

20. Koitabashi, M.: Automorphism groups of generic rational surfaces. J. Algebra 116(1), 130-142 (1988)

21. Lazarsfeld, R.: Positivity in algebraic geometry. II. Ergebnisse der Mathematik und ihrer Grenzgebiete. 3. Folge, vol. 49. A Series of Modern Surveys in Mathematic. Springer, Berlin (2004). Positivity for vector bundles, and multiplier ideals

22. Matsushima, Y.: Sur la structure du groupe d'homéomorphismes analytiques d'une certaine variété kählérienne. Nagoya Math. J. 11, 145-150 (1957)

23. Manin, Yu.I., Hazewinkel, M.: Cubic forms: algebra, geometry, arithmetic. North-Holland, Amsterdam (1974). Translated from Russian by M. Hazewinkel, North-Holland Mathematical Library, vol. 4

24. Nadel, A.M.: Multiplier ideal sheaves and existence of Kähler-Einstein metrics of positive scalar curvature. Proc. Natl. Acad. Sci. USA 86(19), 7299-7300 (1989)

25. Nadel, A.M.: Multiplier ideal sheaves and Kähler-Einstein metrics of positive scalar curvature. Ann. Math. (2) 132(3), 549-596 (1990)

26. Nadel, A.M.: The behavior of multiplier ideal sheaves under morphisms. In: Complex analysis (Wuppertal, 1991), Aspects Math., E17, pp. 205-222. Vieweg, Braunschweig (1991) 
27. Nadel, A.M.: Multiplier ideal sheaves and Futaki's invariant. In: Geometric theory of singular phenomena in partial differential equations (Cortona, 1995), Sympos. Math., vol. XXXVIII, pp. 7-16. Cambridge University Press, Cambridge (1998)

28. Siu, Y.T.: The existence of Kähler-Einstein metrics on manifolds with positive anticanonical line bundle and a suitable finite symmetry group. Ann. Math. (2) 127(3), 585-627 (1988)

29. Tian, G.: On Kähler-Einstein metrics on certain Kähler manifolds with $C_{1}(M)>0$. Invent. Math. 89(2), 225-246 (1987)

30. Tian, G.: On Calabi's conjecture for complex surfaces with positive first Chern class. Invent. Math. 101(1), 101-172 (1990)

31. Tian, G.: Kähler-Einstein metrics with positive scalar curvature. Invent. Math. 130(1), 1-37 (1997)

32. Tian, G.: Canonical metrics in Kähler geometry. Lectures in Mathematics ETH Zürich. Birkhäuser Verlag, Basel (2000). Notes taken by Meike Akveld

33. Tian, G., Yau, S.-T.: Kähler-Einstein metrics on complex surfaces with $C_{1}>0$. Comm. Math. Phys. 112(1), 175-203 (1987)

34. Wiman, A.: Zur Theorie der endlichen Gruppen von birationalen Transformationen in der Ebene. Math. Ann. 48(1-2), 195-240 (1896)

35. Yau, S.T.: Calabi's conjecture and some new results in algebraic geometry. Proc. Natl. Acad. Sci. USA 74(5), 1798-1799 (1977)

36. Yau, S.T.: On the Ricci curvature of a compact Kähler manifold and the complex Monge-Ampère equation. I. Comm. Pure Appl. Math. 31(3), 339-411 (1978) 